\title{
STUDIO MICROSISMICO DEL TERREMOTO \\ DI JENICE DEL 18 MARZO 1953
}

\author{
Francesco Peronaci
}

\section{(PARTE I)}

I. - Il 18 Marzo del 1953 alle ore $19,06^{\mathrm{m}}$ ca. T.M.G. è stato registrato da tutti gli osservatori della rete sismica mondiale un violento terremoto che ha prodotto distruzioni in varie località situate nella regione dell'Anatolia. Particolarmente colpiti risultarono i centri di Jenice, Gonen e Can, in particolare a Jenice tutti gli edifici furono distrutti. Rimandando per le notizie macrosismiche alle pubblicazioni già esistenti sull'argomento, mi limiterò a ricordare che il raggio di percettibilitì delle scosse è stato molto esteso (area di $500.000 \mathrm{~km}^{2}$ ), interessando oltre alla Turchia anche le isole dell'arcipelago greco e la Bulgaria Meridionale. La scossa delle ore 19 ha originato un intenso e lungo periodo sismico, tanto che l'Osservatorio di Istanbul fino al 30 Marzo ha registrato circa 600 repliche, di cui alcune sentite di $3^{\circ}$ grado ad Instanbul. La magnitudo del terremoto, relativamente alla scossa principale, è stata valutata 8 a Berkely, $73 / 4$ a Strasburgo, Pasadena, Tacubaya, 7,5 a Urbanovo e Roma, 7 a Uppsala e De Bilt; assumendo quindi come valore medio della magnitudo 7,5 una valutazione approssimativa ci consente di stabilire che l'energia sviluppata all'ipocentro è risultata dell'ordine di $10^{25} \mathrm{erg}$.

II. - Allo scopo di determinare le coordinate ipocentrali e il tempo origine, è stato applicato un metodo già usato in precedenza con successo nello studio di altri terremoti, limitandomi ai richiami essenziali, ricordo che esso si riduce alla risoluzione di $n$ equazione del tipo

$$
t_{n}=f\left(\lambda_{n}, \varphi_{0}, h_{0}\right)+\frac{\partial f}{\partial \lambda_{0}} \delta \lambda_{0}+\frac{\partial f}{\partial \varphi_{0}} \delta \varphi_{0}+\frac{\partial f}{j h_{0}} \delta h_{0}
$$

ottenute dallo sviluppo in serie fino ai termini di $1^{\circ}$ ordine della funzione $t=f(\lambda, p, h)$.

Nella [1] $p_{0}, \lambda_{0}, t_{0}, h_{0}$ sono $\mathrm{i}$ valori approssimati delle incognite, $\delta \varphi_{0}, \delta \lambda_{0}, \delta t_{0}, \delta \hbar_{0}$, le correzioni da apportare ad esso per ottenere il valore 
più probabile delle incognite stesse: $f\left(\lambda_{0}, \varphi_{0}, h_{0}\right)$ il tempo di propagazione delle $P_{n}$ in corrispondenza della distanza epicentrale considerata, quale risulta dai valori approssimati delle coordinate epicentrali.

Eseguendo i calcoli si trova che indicando con a l'azimut della stazione considerata rispetto all'epicentro, $\frac{\partial f}{\partial \lambda_{0}}$ e $\frac{\partial t}{\partial \varphi_{0}}$ possono essere espressi delle relazioni

$$
\frac{\partial f}{\partial \varphi_{0}}=-\frac{\partial f}{\partial A_{n}} \cos \alpha \quad \frac{\partial f}{\partial \lambda_{0}}-\frac{\partial f}{\partial i_{n}} \cos \varphi_{0} \operatorname{sen} \alpha
$$

per cui la [1] può applicarsi alle varie stazioni nella forma

$$
\begin{gathered}
\delta t_{0}-\cos \varphi_{0} \operatorname{sen} \alpha \frac{\partial f}{\partial \Delta_{n}} \delta \lambda_{0}-\cos \alpha \frac{\partial f}{\partial \Delta_{n}} \delta \varphi_{0}+ \\
+\frac{\partial f}{\partial \varkappa_{0}} \delta h_{0}=P_{n}-\left[t_{0}+f\left(\lambda_{0}, \varphi_{0}, h_{0}\right)\right]
\end{gathered}
$$

ove con $P_{n}$ si è indicato il tempo di arrivo delle longitudinali dirette nella stazione considerata e con $t_{0}+f\left(\lambda_{0}, \varphi_{0}, h_{0}\right)$ la somma dell'ora origine approssimata e del tempo di tragitto. Jeffreys.

Per calcolo della $\frac{\partial f}{\partial \Delta_{n}}$ e $\frac{\partial t}{\partial \tilde{n}_{0}}$ ho impiegato le dromocrone di

Una prima determinazione è stata eseguita utilizzando i dati delle 11 stazioni riportate nella tabella seguente scelte opportunamente sia per l'elevato scorrimento che per la loro particolare posizione geografica. In particolare ho ritenuto di trascurare le stazioni che comportavano distanze inferiori ai $1500 \mathrm{~km}$ allo scopo di utilizzare tragitti sul cui tempo non influissero eccessivamente le prime stratificazioni della crosta terrestre. Ho ritenuto cle ciò fosse necessario in quanto non solo non esistono dromocrone per terremoto con provenienza dal bacino orientale del Mediterraneo, ma anche perchè altri ricercatori hanno sospettato delle anomalie sulla propagazione delle onde sismiche per terremoti con tale provenienza.

Assunto come epicentro provvisorio quello fornito dal B.C.I.S. $\varphi_{0}-40^{\circ} 6^{\prime} \mathrm{N} ; \lambda_{0}=27^{\circ} 18^{\prime} \mathrm{E}$, e per tempo origine provvisorio quello alcolato in base ai tempi di tragitto per le varie stazioni yer $h=0$. $\left(t_{0}-19^{\mathrm{h}} 06^{\prime} 12^{\prime \prime}\right)$ ho ottenuto impiegando gli elementi della tabella 1 le seguenti correziomi da apportare ai dati ipocentrali provvisori: $\delta q_{0}=-0^{\circ}, 00493599, \delta \lambda_{0}=+0^{\circ}, 0271650, \delta t_{0}=-1,5973 \mathrm{sec}$ 
$\delta h_{0}=-10 \mathrm{~km}$. I calcoli sono stati eseguiti in coordinate geocentriche, mentre sia $\delta \varphi_{0}$ che $\delta \hat{\Lambda}_{0}$ sono espresse in unità gradi geografici.

Tabella 1

\begin{tabular}{|c|c|c|c|c|c|}
\hline Stazioni & $\begin{array}{c}\text { Distanza } \\
\mathrm{K} \mathrm{m} .\end{array}$ & $P n$ & $\begin{array}{c}f\left(\varphi_{0}, \gamma_{0}, h_{0}\right) \\
\text { per } h=0\end{array}$ & $t_{0}$ & $\begin{array}{l}P n-\iota_{0} \\
+f\left(\varphi_{0}, \lambda_{0}, h_{0}\right)\end{array}$ \\
\hline Stocearda & 1726 & $09 \mathrm{~m} 53 \mathrm{~s}, 5$ & $3 \mathrm{~m} 42 \mathrm{~s}, 0$ & $06 \mathrm{~m} 11=, 0$ & $-0 \mathrm{~s}, 6$ \\
\hline Kew . . & 2468 & 1121,1 & 459,6 & $06 \quad 12,5$ & $+0,4$ \\
\hline Jena . . & 1713 & 0952,5 & 340,4 & $06 \quad 12,1$ & 0,0 \\
\hline Algeri. . . & 2141 & 1041,0 & 428,7 & $06 \quad 12,3$ & $+0,2$ \\
\hline Strasburgo . & 1814 & 1004,2 & 352,0 & $06 \quad 12,2$ & $\dashv-0,1$ \\
\hline Uppsala . . & 2298 & 1055,8 & 444,1 & 0611,7 & $-0,4$ \\
\hline De Bilt & 2154 & 1042,2 & $\begin{array}{lll}429 & 9\end{array}$ & $06 \quad 12,3$ & $+0,2$ \\
\hline Neuchatel. & 1809 & 1003,0 & 351,1 & 0611,9 & $-0,2$ \\
\hline Göttingen . & 1843 & 1007,9 & 355,5 & $06 \quad 12,4$ & $+0,3$ \\
\hline 'Iortosa & 2265 & 1053,0 & 441,1 & 0611,9 & $-0,2$ \\
\hline Kiruna & 3117 & 1207,1 & 554,9 & $06 \quad 12,2$ & $+0,1$ \\
\hline
\end{tabular}

Tenendo presente che l'asse verticale è scelto positivo verso il centro della Terra, il calcolo porterebbe l'ipocentro al disopra della superficie terrestre. Valutato l'errore quadratico medio per la profondità ho ottenuto 土 $15 \mathrm{~km}$ quindi lıo supposto che la profondità ipocentrale fosse ralmente piccola da non poter essere valutata se non con l'impiego di stazioni molto prossime all'ipocentro.

Mantenendo l'ipotesi di ipocentro superficiale ho posto nella [1'] $h_{0}=0$, e ho rieseguito i calcoli con le 11 stazioni già impiegate, ottenendo con due approssimazioni successive $\mathrm{i}$ seguenti dati più probabili della posizione dell'epicentro e del tempo origine (anche qui $\varphi_{0}$ e $\lambda_{0}$ sono espressi in coordinate geografiche).

$$
\begin{aligned}
& \varphi_{0}=40^{\circ} 07^{\prime} 09^{\prime \prime} \mathrm{N} \pm 2^{\prime} 20^{\prime \prime} \mathrm{N} . \\
& \lambda_{0}=27^{\circ} 17^{\prime} 29^{\prime \prime} \mathrm{E} \pm 30^{\prime} 0^{\prime} \mathrm{E} . \\
& t_{0}=19^{\prime \prime} 06^{\prime} 12^{\prime \prime}, 3 \pm 0,6^{\text {s }} \text { T.M.G. }
\end{aligned}
$$


Ho ritenuto interessante eseguire una seconda determinazione della posizione dell'ipocentro e del tempo origine impiegando i dati di stazioni più prossime all'epicentro ed in particolare di Vienna, Roma, Bologna, Messina, Trieste, Salò, Padova, Reggio Calabria, Budapest, Urbanovo tutte incluse tra 1000 e $1500 \mathrm{~km}$. Per i calcoli mi sono questa volta servito della dromocrona delle $P$ n calcolata da Di Filippo-Marcelli valida per l'Italia Centrale per una profondità di $5 \mathrm{~km}$ in quanto più prossima alla zona studiata e della dromocrona di Jeffreys per $h=33 \mathrm{~km}$. Il tempo origine medio quale si calcola facendo la differenza tra tempo di registrazione e tempo di tragitto è risultato in base alla dromocrona di Di Filippo-Marcelli di $19^{\mathrm{n}} 06^{\mathrm{m}} 12^{\mathrm{s}}, 0$ con un massimo scarto di 0,8 sec per là stazione di Salò.

Eseguiti i calcoli ho ottenuto con 2 approssimazioni successive

$$
\begin{gathered}
\varphi_{0}=40^{\circ} 07^{\prime} 15^{\prime \prime} \pm 3^{\prime}, 4 \mathrm{~N} \quad \lambda_{0}=27^{\circ} 17^{\prime} 59^{\prime \prime} \pm 3^{\prime}, 4 \mathrm{E} \\
t_{0}=19^{\mathrm{h}} 06^{\prime} 12^{\prime \prime}, 1 \pm 0^{\mathrm{s}}, 7
\end{gathered}
$$

con una correzione per la profondità di $-7 \mathrm{~km} \pm 10$ che riporterebbe l'ipocentro in superficie dato che la correzione va applicata a $5 \mathrm{~km}$ di supposta profondità ipocentrale. L'accordo tra le due determinazioni ̀̀ veramente sorprendente e fa sospettare che non si possa parlare per le onde spaziali di anomalie di propagazione nei tragitti che interessano la parte meridionale della penisola Balcanica e il Mediterraneo Orientale, almeno quando si tratti di tempi di tragitto corrispondenti a distanze non inferiori ai $1000 \mathrm{~km}$ come nel caso delle stazioni impiegate. Di questo fatto si ha una conferma osservando gli scarti $P \eta-t+f\left(\varphi_{0}, \lambda, h_{0}\right)$, che per le varie stazioni sono: Vienna -0 s, 1 ; Roma $+0,7$; Bologna $+0,5$; Messina $+0,4$; Trieste +0.6 ; Salò $-0,8$; Padova $-0,6$; Reggio C. $+0,5$; Budapest $-0,4$; Urbanovo - 0,7 .

Essendo durante il corso della ricerca giunte le registrazioni ottenute dalle stazioni rumene, che con Atene sono le più vicine all'epicentro, ho voluto rieseguire il calcolo dell'ipocentro impiegando i dati di stazioni che comportassero tragitti esclusivamente continentali e interessanti la zona dei Balcani e le cui distanze epicentrali non superassero i $1200 \mathrm{~km}$. A questo scopo furono impiegate le stazioni di Bucarest, Campolungh, Foxani, Bacau, Jasi, Budapest, Zagabria, Urbanovo, Vienna. Particolarmente laboriosa è stata la scelta della dromocrona da adoperare; infatti tentativi eseguiti sia impiegando le dromocrone di Caloi per il Cansiglio, che quelle di Mohorovicic per i Balcani, non hanno portato a risultati attentibili, essendo l'epicentro microsismico risultato troppo lontano 
dall'epicentro macrosismico. La ragione di ciò è ad esempio da ricercarsi nel caso della dromocrona di Caloi, al fatto che essa oltre ad essere calcolata per una profondità di $17 \mathrm{~km}$, si riferisce ad una zona ove la potenza dello strato del granito raggiunge i $35-10 \mathrm{~km}$, valore che è nettamente superiore a quello che presumibilmente si deve attribuire alla zona studiata. Come ultimo tentativo ho impiegato le dromocrone ricavate per l'Italia centrale da Di Filippo-Marcelli e la dromocrona per $h=33 \mathrm{~km}$ di Jeffreys e con due approssimazioni successive ho ottenuto i seguenti valori più probabili delle correzioni da apportare ai valori provvisori relle incognite:

$$
\begin{array}{ll}
\delta \varphi_{0}=-0^{\circ}, 0662 & \delta \lambda_{0}=-0^{\circ}, 00826 \\
\delta h_{0}=-4 \mathrm{~km} & \delta t_{0}=-0,922 \mathrm{sec}
\end{array}
$$

per cui tenendo presente che i valori di partenza erano

$$
\begin{array}{ll}
\varphi_{0}=40^{\circ} 07^{\prime} 09^{\prime \prime} \mathrm{N} & \lambda_{0}=27^{\circ} 17^{\prime} 29^{\prime \prime} \mathrm{E} \\
t_{0}=19^{\mathrm{h}} 06^{\mathrm{m} 1} 12^{\mathrm{s}}, 3 & h_{\mathrm{o}}=5 \mathrm{~km}
\end{array}
$$

i risultati dei calcoli confermano ancora la superficialità del terremoto o i dati epicentrali che erano stati ricavati impiegando le stazioni lontane. Per quanto riguarda gli scarti tra i tempi di registrazione osservati e i calcolati per le varie stazioni, avevo ottenuto impiegando le dromocrone di Di Filippo-Marcelli: Bucarest $+0^{\text {s }}, 6$; Campolungh 0,0; Foxani + 0,7; Bacau - 0,2; Jasi - 0,9; Budapest-0,6; Zagabria 0,0; Urbanovo-0,8; Vienna - 0,3 ; cioè valori che possono tranquillamente attribuirsi agli errori di osservazione.

Concludendo possiamo assumere come coordinate dell'epicentro quelle ricavate con le stazioni lontane e affermare che la profondità ipocentrale è molto piccola, e probabilmente compresa nei primi $5 \mathrm{Km}$ di profondità. L'assenza di materiale di osservazione relativo a stazioni molto prossime all'epicentro non consente una determinazione più rigorosa della profondità ipocentrale, per cui almeno per adesso dovremo limitarci a tale affermazione rimandando ogni ulteriore conferma al proseguo della ricerca.

III. - Sono quindi passato a calcolare le dromocrone delle onde spaziali $P u$ ed $S n$ fino a $3500 \mathrm{~km}$; come è noto tali dromocrone possono essere senza errore sensibile, approssimate a rette solo fino a $1000 \mathrm{~km}$, per cui oltre tale distanza è necessario approssimarle ad una curva. Un primo tentativo eseguito con curva di $2^{\circ}$ grado, pur fornendo risultati discreti non è stato ritenuto soddisfacente, per cui ho preferito eseguire 
l'approssimazione delle dromocrone sperimentali con una curva di $3^{\circ}$ grado del tipo

$$
t=\alpha+\beta \Delta+\gamma \Delta^{3}+\delta \Delta^{2}
$$

nella quale $t$ è il tempo di tragitto, $\Delta$ la distanza epicentrale, e $\alpha, \beta, \gamma, \delta$ coefficienti indipendenti da $\Delta$ da determinare usufruendo dei dati sperimentali relativi ad un numero sufficiente di stazioni di osservazione, con il metodo dei numeri quadrati.

La [2] è stata applicata alle 15 stazioni comprese tra 600 e $3000 \mathrm{~km}$ riportate nella tabella 2. Per rendere meno laboriosi i calcoli che tra l'altro comportano l'impiego di numeri con cifre comprese tra 15 e 20 , ho proceduto nel seguente modo: scritte le 15 equazioni per ordine di distanza epicentrale crescente, ho dedotto per differenza 14 equazioni nelle sole $\beta, \gamma, \delta$ che ho risolto con il metodo dei minimi quadrati ottenendo i seguenti valori provvisori di $\beta, \gamma, \delta$ :

$$
\begin{aligned}
& \beta=+0,117646216911 \\
& \gamma=+0,635330334151 \cdot 10^{-5} \\
& \delta=-0,280260316705 \cdot 10^{-\gamma} .
\end{aligned}
$$

In base a tali valori, che ho sostituito nelle [2], ho ricavato il valore di $a$ ottenendo $\alpha=+13,64173896$ che introdotto nelle [2] ha consentito di ottenere 15 equazioni nelle $\beta, \gamma, \delta$ che ho risolto sempre con il metodo dei minimi quadrati. In definitiva l'equazione della dromocrona più probabile delle $P n$ è data dalla equazione

$$
t=13,6417+0,119351 \Delta+0,46451 \cdot 10^{-5} \Delta^{2}-0,238965.10^{-8} \Delta^{3}
$$

con errore quadratico medio $\varepsilon= \pm 0,83015$. Gli scarti tra valori calcolati e valori osservati sono riportati nell'ultima colonna nella tabella 2 , e ci mostrano come la curva calcolata approssimi la dromocrona sperimentale entro i limiti degli errori di osservazione.

IV. - Impiegando stazioni con distanze non superiori ai $1250 \mathrm{~km}$ ho calcolato la dromocrona delle $P n$ approssimandola ad una retta; i dati sperimentali sono quelli della tabella 3 , ove anche sono stati riportati gli scarti della dromocrona più probabile dai tempi rilevati dai sismogrammi. 
Tabella 2

\begin{tabular}{|c|c|c|c|c|c|}
\hline$\sqrt{2}+2$ & Stazioni & & $\Delta / \mathrm{Km}$ & $\begin{array}{c}t_{P n} \\
\text { (osservato) }\end{array}$ & $\Delta t$ \\
\hline Campolungh & . . . & ...... & 601 & $1 \mathrm{~m} 26 \mathrm{~s}, 2$ & $+0,31$ see \\
\hline Bacaul. . & .... & ...... & 715 & 139,9 & $+0,36$ \\
\hline Messina & $\ldots$ & ...... & 1036 & $2 \quad 19,5$ & $+0,30$ \\
\hline Zagabria & . . . . & ..... & 1115 & 228,9 & $+0,76$ \\
\hline Roma . & $\ldots$ & ..... & 1257 & 247,0 & $-0,58$ \\
\hline Padova & .... & . . . & 1389 & 301,6 & $+0,39$ \\
\hline Praga & $\ldots$ & $\ldots \ldots$ & 1492 & $\begin{array}{lll}3 & 13,4\end{array}$ & $+0,68$ \\
\hline Kebb & . . . . &.... & 1609 & 326,5 & $+1,26$ \\
\hline Jena . & . . . & . . . . & 1711 & 340,2 & $-0,73$ \\
\hline Strasburgo & .... & ..... & 1811 & 351,9 & $-1,08$ \\
\hline Algeri & $\ldots$ & $\ldots$ & 2140 & 428,7 & $-1,69$ \\
\hline Uppsala . & . . . . & . . . . & 2296 & 443,1 & $-0,15$ \\
\hline Kew. . &... & . . & 2468 & $4 \quad 59,8$ & $+0,77$ \\
\hline Almeria &... & . & 2607 & $\begin{array}{lll}5 & 13,7\end{array}$ & $+0,40$ \\
\hline Malaga & .... & . . . . . & 2778 & $\begin{array}{lll}529,7\end{array}$ & $+0,19$ \\
\hline
\end{tabular}

La dromocrona più probabile delle $P n$ fino a $1250 \mathrm{~km}$ è espressa dall'equazione

$$
t=-\frac{\Lambda}{8,168 \pm 0,036}+12^{\mathrm{s}, 81} \pm 0^{\mathrm{s}, 79} .
$$

Per cui la velocità media alla base della crosta terrestre risulta di 8,17 $\mathrm{km} / \mathrm{sec}$. Questo valore è molto prossimo a $8,19 \pm 0,0+$ di Di FilippoMarcelli per l'Italia centrale (Gran Sasso) e all'8,14 Karnik per i Balcani.

Nell'ultima colonna della tabella sono riportati gli scarti rispetto alla curva di $3^{\circ}$ ordine, dal loro esame si nota che per distanze fino a $1200 \mathrm{~km}$ si deve accordare una leggera preferenza alla retta in quanto essa approssima meglio i valori sperimentali.

Ho ritenuto utile eseguire un confronto della dromocrona calcolata con le dromocrone per la superficie calcolate da Gutenberg, Jeffreys (1948) e rla Di Filippo-Marcelli (per una profondità (li $5 \mathrm{~km}$ ) (tabella 4); 
Tabella 3

\begin{tabular}{|c|c|c|c|c|}
\hline Stazioni & $\Delta / \mathrm{Km}$ & $t_{P_{n}}$ & $\begin{array}{c}\Delta t \\
\text { (dalla retta) }\end{array}$ & $\begin{array}{c}\Delta t \\
\text { (dalla curva } \\
\text { di } 3^{\circ} \text { grado) }\end{array}$ \\
\hline Bucarest & 489 & $1 \mathrm{~m} 13 \mathrm{~s}, 1$ & $-0,5$ & $-0,2$ \\
\hline Campolungh & 601 & 126,2 & 0,0 & $-0,3$ \\
\hline Foxani & 621 & 129,3 & $-0,6$ & 0,3 \\
\hline Bacau & 715 & 139,9 & $+0,3$ & $+0,4$ \\
\hline . . . & 788 & 148,2 & $+0,9$ & $+1,0$ \\
\hline Reggio Cal.. . . & 1030 & 218,8 & $-0,2$ & $+0,1$ \\
\hline . . . . & 1036 & 219,5 & $-0,1$ & $+0,3$ \\
\hline Budapest & 1051 & 220,5 & $+0,7$ & \\
\hline Zagabria ... & 1115 & 228,9 & $+0,1$ & $+0,8$ \\
\hline Urbanovo & 1128 & 229,6 & $+1,0$ & $+1,2$ \\
\hline Vienna & 1254 & 245,6 & $+0,6$ & $+0,7$ \\
\hline Roina . . . . . & 1257 & 247,0 & $-0,6$ & $-0,6$ \\
\hline Trieste. . . . . & 1264 & 248,8 & $-1,6$ & $-1,5$ \\
\hline
\end{tabular}

si nota una notevole coincidenza almeno fino a $1000 \mathrm{~km}$, coincidenza che comporta l'ipotesi che la profondità ipocentrale del terremoto in esame sia molto piccola.

V. - Utilizzando i dati delle seguenti stazioni di osservazione ho tentato di approssimare la domocrona delle sn con una curva di terzo ordine del tipo

$$
t=\alpha+\beta \Delta+\gamma \Delta^{2}+\delta \Delta^{3} .
$$

Il risultato dei calcoli ha indicato come curva più probabile la:

$$
t=11,6560+0,23121 \triangle+0,736171.10^{-.8} \Delta^{2}-0,314557.10^{-8} \Delta^{3}
$$

con un errore quadratico medio $\varepsilon= \pm 0,872214$.

Gli scarti tra valori osservati e valori calcolari relativi ai tempi di propagazione delle sn sono riportati nell'ultima colonna dei dati di osservazione impiegati per costruire la dromocrona. 
Tabella 4

\begin{tabular}{|c|c|c|c|c|}
\hline$\Delta^{0}$ & Gutenberg & Jeffreys & $\begin{array}{l}\text { Di Filippo- } \\
\text { Marcelli }\end{array}$ & Terr. Turchia \\
\hline 4 & -- & $\operatorname{lm}_{03}, 9$ & $1 \mathrm{~m} 07 \mathrm{~s}, 0$ & $1 \mathrm{~m} 07 \mathrm{~s} ; \mathrm{l}$ \\
\hline B & -- & 132,2 & 134,3 & 134,2 \\
\hline 8 & -- & 200,3 & 201,3 & 201,4 \\
\hline 10 & $2 \mathrm{~m} 27 \mathrm{~s}$ & 228,0 & 228,6 & 228,7 \\
\hline 12 & 255 & 255,3 & 265,2 & 255,3 \\
\hline 14 & 323 & $\begin{array}{lll}3 & 21 & 9\end{array}$ & --- & 321,5 \\
\hline 16 & 349 & 348,0 & $-\cdots-$ & 347,0 \\
\hline 18 & 413 & 413,2 & --- & 411,8 \\
\hline 20 & 435 & 437,0 & $-\ldots$ & 435,6 \\
\hline 22 & 457 & 457,5 & --- & 458,2 \\
\hline 24 & 517 & 517,1 & $-\cdots$ & 519,6 \\
\hline
\end{tabular}

I valori di tali scarti, pur essendo in generale maggiori di quelli relativi alle $P n$, sono contenuti in limiti ragionevoli e quasi certamente imputabili alla difficoltà di rilevare l'inizio delle Sn dai sismogrammi; difficoltà principalmente dovuta al fatto che gli inizi delle $S n$ sono per queste distanze epicentrali particolarmente deboli in quanto le distanze stesse risultano comprese nella zona d'ombra originata dalla presenza della astenosfera.

Anche per le onde trasversali dirette ho eseguito il tentativo di approssimazione della dromocrona fino a $1200 \mathrm{~km}$ ad una retta e impiegando le stazioni di Atene, Campolungh, Foxani, Bacau, Jasi, Reggio C., Messina, Budapest, Zagabria, Hurbanovo, Vienna, Roma ho ottenuto

$$
t=\frac{\Delta}{4,40 \pm 0,01}-+12,82 \pm 0,67
$$

quindi la velocità media delle trasversali alla base della superficie di Mohorovicic è $4,40+0,01 \mathrm{~km} / \mathrm{sec}$. Gli scarti tra tempi osservati e tempi calcolati sono, nell'ordine di come sono state scritte le stazioni: $-0^{5}, 22$; $-0,17 ;-0,02 ;-0,34 ;+1,36 ;-0,29 ;-0,03 ;-0,01 ;+0,74 ;-1,20$; $+0,46 ;-0,26$. L'errore quadratico medio è risultato $\varepsilon= \pm 0,66406$. 
Tabella $\tilde{\mathbf{s}}$

\begin{tabular}{|c|c|c|c|c|}
\hline & Stazioni & $\begin{array}{c}\text { Distanze } \\
\text { (kim.) }\end{array}$ & $t_{\text {(osservato) }}$ & $\Delta l$ \\
\hline Campolungh & $\ldots \ldots$ & 601 & $2 \mathrm{~m} 29 \mathrm{~s}, 7$ & $\tau^{-} 0,547$ \\
\hline Bacau & $\ldots \ldots$ & 715 & 255,8 & $\therefore \quad 0,26$ \\
\hline Messina & . . . & 1036 & 408,5 & ㅏ 0,07 \\
\hline Zagabria & $\ldots \ldots$ & 1115 & 425,7 & $+0,20$ \\
\hline Roma. . & $\ldots \ldots$ & 1257 & 459,0 & $--1,75$ \\
\hline Bologna & $\ldots \ldots$ & 1400 & 529,6 & $\ldots-1,45$ \\
\hline Praga . . & $\ldots \ldots$ & 1492 & 547,8 & $-0,22$ \\
\hline Pavia. & $\ldots \ldots$ & 1.584 & 606,2 & $+0,98$ \\
\hline Jena & $\ldots \ldots$ & 1711 & $\begin{array}{lll}6 & 31 & , 7\end{array}$ & $+1,93$ \\
\hline Staccarcla & $\ldots \ldots$ & 1724 & 635,2 & $+1,09$ \\
\hline Strasburgo & . . . . . . & 1811 & 654,4 & $-0,31$ \\
\hline Gottinga & $\ldots \ldots$ & 1841 & 659,7 & $+0,46$ \\
\hline Copenaghen & . . . & 2046 & 741,7 & $-0,88$ \\
\hline Algeri . & $\ldots \ldots$ & 2141 & 800,7 & $-1,53$ \\
\hline Uppsala & $\ldots \ldots$ & 2296 & 826,9 & $+1,54$ \\
\hline Kew & . . . . . & 2468 & $90 \mathrm{i}, 0$ & $-1,59$ \\
\hline Almeria & ........ & 2607 & 922,7 & $+0,87$ \\
\hline
\end{tabular}

Anche in questo caso ho osservato che fino a $1200 \mathrm{~km}$ si deve preferire la dromocrona rettilined alla curva di terzo ordine.

Roma - Istituto Nazionale di Geofisica - Dicembre 1955.

\section{RIASSUNTO}

$\Pi$ presente lavoro è la prima parte di uno studio eseguito sul terremoto di Jenice del 18 Marzo 1953. In esso vengono calcolate le coordinate epicentrali, e le dromocrone più probabili delle $P n$ e delle $S n$ valide fino a $3000 \mathrm{~km}$. 


\section{$S U M M A R Y$}

The present paper is the first part of a study on the earthquake of Jenice (1953, March 18). The epicentral coordinates and time-curves of $P_{n}$ and Sn phases (till about $3000 \mathrm{kms}$ ) has been calculated.

\section{B I B L I O G R A F I A}

KEтIN, I.. e RoesLI, F., Makroseimische Untersuchungen über das nordwestanalolische Beben von 18 März 1953. Eclogae Geologicae Helvetiae, Vol. 46, n. 2, 1953.

Caloi, P., e Peronaci, F., Il terremolo del Turkestan del 2 Nov. 19:6. Annali di Geofisica, Vol. I, n. 2, 1948.

Di Filippo, D., e Marcelli, L., Uno sludio del lerremolo del Gran Sasso d'Ilalia del 5 Setlembre 19.50. Annali di Geofisica, Vol. IV, n. 2, 1951.

Calol, P., Tempi di tragitlo per terremoti ad origine ticina. La Ricerca Scientifica, Vol. X, n. 5, 1939.

Girlanda, A., Il terremoto di Hokkaido del $\neq$ Marzo 1952. Annali di Geofisica, Vol. VI, n. 2, 1953.

Gutenber(, B., e Richter, C. F., On seismic waves (First Paper). Gerl. Beitr. z. Geoph., Band 43, 1935.

Jeffreys, H., e Bullen, K. E., Seismological Tables. British Assoc. for Advancement of Science, London, 1948. 